\title{
IncRNA SNHG22 sponges miR-128-3p to promote the progression of colorectal cancer by upregulating E2F3
}

\author{
JIANNING YAO* , CHUNFENG WANG ${ }^{*}$, XUYANG DONG, YANZHEN ZHANG, \\ YANLE LI, HAINING ZHOU and LIANFENG ZHANG
}

Department of Gastroenterology, The First Affiliated Hospital, Zhengzhou University, Zhengzhou, Henan 450052, P.R. China

Received November 15, 2020; Accepted June 14, 2021

DOI: 10.3892/ijo.2021.5251

\begin{abstract}
The long non-coding RNA (lncRNA) small nucleolar RNA host gene 22 (SNHG22) has been reported as a crucial regulator in several types of human cancer. The present study evaluated the function and mechanism of SNHG22 in colorectal cancer (CRC) progression. SNHG22 expression was detected in colorectal adenoma, CRC tumor tissues (TTs) and adjacent non-cancerous tissues (ANTs) using reverse transcription-quantitative PCR (RT-qPCR). The biological behaviors of SNHG22 in CRC cell lines were explored in vitro using Cell Counting Kit-8, flow cytometry, wound scratch assay and Transwell assay, and in vivo using a nude mouse xenograft model. The interaction between SNHG22 and microRNA-128-3p (miR-128-3p), and the target genes of miR-128-3p were explored using online tools, RT-qPCR, western blotting and a dual-luciferase reporter assay. The present study revealed that SNHG22 expression was most highly expressed in TTs followed by adenoma tissues and ANTs. In addition, high SNHG22 expression levels were significantly associated with advanced clinicopathological factors and worse survival in patients with CRC. SNHG22
\end{abstract}

Correspondence to: $\mathrm{Dr}$ Lianfeng Zhang, Department of Gastroenterology, The First Affiliated Hospital, Zhengzhou University, 1 Jianshe Dong Road, Zhengzhou, Henan 450052, P.R. China

E-mail: 1900932217@qq.com

Abbreviations: CRC, colorectal cancer; EOC, epithelial ovarian carcinoma; PTC, papillary thyroid cancer; lncRNA, long non-coding RNA; snoRNA, small nucleolar RNA; SNHG, small nucleolar RNA host gene; siRNA, small interfering RNA; UTR, untranslated region; mut, mutant; RIP, RNA immunoprecipitation; CCK-8, Cell Counting Kit-8; TT, tumor tissue; ANT, adjacent non-cancerous tissue; TCGA, The Cancer Genome Atlas; DFS, disease-free survival; OS, overall survival; ceRNA, competing endogenous RNA; HR, hazard ratio; CI, confidence interval

*Contributed equally

Key words: small nucleolar RNA host gene 22, E2F transcription factor 3, microRNA-128-3p, colorectal cancer knockdown markedly inhibited CRC cell proliferation, apoptosis resistance, migration and invasion in vitro, and hindered tumor growth in vivo. The mechanistic study revealed that SNHG22 bound to miR-128-3p and attenuated its inhibitory effects on E2F transcription factor 3 (E2F3) expression levels and activity. Rescue experiments demonstrated that inhibiting miR-128-3p or upregulating E2F3 offset the effects of SNHG22 knockdown on CRC cells. The present findings support the existence of an interactive regulatory network involving SNHG22, miR-128-3p and E2F3 in CRC cell lines, indicating that the SNHG22/miR-128-3p/E2F3 axis may be considered a novel diagnostic and therapeutic target in CRC.

\section{Introduction}

Colorectal cancer (CRC) represents one of the most common malignancies worldwide. Every year, $>1.3$ million people are diagnosed with CRC globally, of which $\sim 0.7$ million succumb to this disease (1). Only $\sim 50 \%$ of patients with CRC survive for 5 years after diagnosis in Europe (2), despite some improvements being made in early diagnosis and systemic therapies. The pathogenesis and progression of CRC is a complex process, and the potential mechanism remains unclear.

Long non-coding RNAs (lncRNAs) are a class of non-encoding RNAs, which are $>200$ nucleotides long. Recent research has indicated that numerous lncRNAs serve an important role in the majority of physiological and pathological processes, including embryonic stem cell self-renewal, carcinogenesis and cancer metastasis $(3,4)$. In addition, lncRNAs function as oncogenes or tumor suppressor genes, which participate in CRC tumorigenesis and progression. For example, Xu et al (5) demonstrated that the lncRNA microRNA (miRNA/miR)-17-92a-1 cluster host gene (MIR17HG) drove tumor development and metastasis in CRC cells by enhancing the expression of $\mathrm{NF}-\kappa \mathrm{B} / \mathrm{RELA}$. In addition, $\mathrm{HOX}$ transcript antisense RNA has been revealed to enhance CRC cell migration and drug resistance via a miR-203a-3p-dependent Wnt/ $\beta$-catenin signaling pathway (6). As an oncogene, the lncRNA $H 19$ has been shown to accelerate cell proliferation and metastasis in CRC by acting on Wnt signaling (7).

Small nucleolar RNAs (snoRNAs) are a subgroup of ncRNAs that are 60-300 nucleotides in length, which contribute to tumorigenesis and metastasis in diverse types of human cancer. Most snoRNAs are encoded in the introns of snoRNA 
host genes (SNHGs) (8). Specifically, there are numerous SNHGs associated with CRC carcinogenesis and progression; for example, SNHG7 has been shown to be overexpressed in CRC tumor tissues (TTs) compared with its expression in adjacent non-cancerous tissues (ANTs). This high expression has been revealed to be related to aggressive pathological characteristics, such as tumor size, tumor-node-metastasis (TNM) stage, distant metastasis and poor survival $(9,10)$. Located on chromosome 8q21.1, SNHG22 is 2,157 nucleotides long (11). Previous studies have reported that $S N H G 22$ contributes to cell growth, migration, invasion and chemotherapy resistance in epithelial ovarian carcinoma (EOC), papillary thyroid cancer (PTC) and breast cancer (12-14). Notably, in these three types of cancer, SNHG22 expression was revealed to be upregulated in TTs compared with that in ANTs, thus indicating its association with poor prognosis. To the best of our knowledge, the biological role and expression patterns of SNHG22 have not been examined in human CRC.

The present study investigated SNHG22 expression in adenoma and CRC tissues, and evaluated its clinical significance. Furthermore, the biological behaviors of SNHG22 were explored in CRC cell lines in vitro and in vivo. The present study also investigated the mechanisms underlying the pro-oncogenic effects of SNHG22 on CRC.

\section{Materials and methods}

Tissue samples and cell lines. A total of 93 paired CRC TTs and matched ANTs were collected from patients who had undergone surgery between January 2012 and October 2012 (Zhengzhou cohort). Additional fresh specimens were collected from patients with colorectal adenoma (CRA) $(n=33)$ who had undergone colonoscopy. The CRA or CRC diagnoses were based on histopathological evaluation using the 7th edition of the American Joint Committee on Cancer staging system (15). All specimens were quickly snap-frozen in liquid nitrogen and stored at $-80^{\circ} \mathrm{C}$ until required. The present study was approved by the Ethics Committee of Zhengzhou University (approval no. 2011110402; Zhengzhou, China). Patients with any history of other types of cancer, and who had received preoperative radiotherapy or chemotherapy were excluded. The clinical characteristics of all patients are listed in Table SI.

Human colon cancer cell lines (Caco2, LS174T, LoVo, SW480, SW620) and a CRC cell line (HT-29) were all obtained from the Cell Bank of the Chinese Academy of Sciences. The cells were maintained in RPMI-1640 medium (Gibco; Thermo Fisher Scientific, Inc.) containing 10\% fetal bovine serum (FBS; Gibco; Thermo Fisher Scientific, Inc.) in a humidified incubator $\left(37^{\circ} \mathrm{C}, 5 \% \mathrm{CO}_{2}\right)$. The $\mathrm{FHC}$ human normal colon epithelial cell line was purchased from Mingzhou Company (cat. no. MZ-0713) and was cultured in 90\% Dulbecco's modified Eagle's medium (DMEM; Gibco; Thermo Fisher Scientific, Inc.) supplemented with 20\% FBS. All cells tested negative for mycoplasma contamination and this result was verified by short tandem repeat fingerprinting before use.

Cell transfection. Small interfering RNAs (siRNAs) against SNHG22 (si\#1 and si\#2) or E2F transcription factor 3 (E2F3) (siE2F3), and the overexpression vectors pcDNA3.1/Control (Vector), pcDNA3.1/SNHG22 (SNHG22) and pcDNA3.1/E2F3
(E2F3) were purchased from Shanghai GenePharma Co., Ltd.. A non-targeting sequence was used as the negative control (siNC; Shanghai GenePharma Co. Ltd.). The miRNA mimics, inhibitor and negative controls (NC mimic and $\mathrm{NC}$ inhibitor) were purchased from Guangzhou RiboBio Co., Ltd.. For the in vivo experiments, vectors containing short hairpin RNAs (shRNAs) targeting SNHG22 (shSNHG22, i.e., sh\#1 and sh\#2) or a non-targeting sequence (shNC) were subcloned into Lv5 lentiviruses (Shanghai GenePharma Co., Ltd.) and infected into LoVo cells to generate $\mathrm{Lv}$-sh\#1 and Lv-sh\#2. For LoVo cells infection, cells $\left(5 \times 10^{4}\right.$ cell/well) were cultured for $24 \mathrm{~h}$, and then recombinant lentivirus in serum-free growth medium was added at a multiplicity of infection of 50 at $37^{\circ} \mathrm{C}$ for 2 days. For transient transfection, Caco2, LS174T and LoVo cells $\left(3 \times 10^{4}\right.$ cell/well) in 6 -well plates were transfected with vectors/sequences at a concentration of $20 \mu \mathrm{g} / \mathrm{ml}$ at $37^{\circ} \mathrm{C}$. After culturing for $24 \mathrm{~h}$, cells were harvested for subsequent experiments. Transfection experiments were conducted with Lipofectamine ${ }^{\circledR} 3000$ transfection reagent (Invitrogen; Thermo Fisher Scientific, Inc.) according to the manufacturer's instructions. All the aforementioned sequences are shown in Table SII.

Cell proliferation. Cell proliferation was detected using Cell Counting Kit-8 (CCK-8; Beyotime Institute of Biotechnology) according to the manufacturer's instructions. The cells were transfected with the indicated vectors or sequences, and then cultured in a 96-well plate for 1, 2, 3 and 4 days after transfection. Subsequently, $\sim 10 \mu \mathrm{l}$ CCK- 8 reagent was added per well, and incubated at $37^{\circ} \mathrm{C}$ for $2 \mathrm{~h}$ in a $5 \% \mathrm{CO}_{2}$ humidified chamber. The absorbance was measured at $490 \mathrm{~nm}$ in each well using a microplate reader (Bio-Rad Laboratories, Inc.). The experiment was repeated at least three times.

Flow cytometry. Cell cycle distribution was detected using flow cytometry. After the CRC cells had been harvested and washed, cells were detected with a Cell Cycle and Apoptosis Analysis Kit (cat. no. C1052M; Beyotime Institute of Biotechnology) according to the manufacturer's protocol. Apoptosis was detected using an Annexin V-fluorescein isothiocyanate (FITC)/propidium iodide (PI)-Apoptosis Detection kit [MultiSciences (Lianke) Biotech Co., Ltd.]. CRC cells $\left(5 \times 10^{4} /\right.$ well $)$ were seeded and resuspended in 12-well plates, and Annexin V-FITC (5 $\mu \mathrm{l} /$ well) and PI (100 $\mu \mathrm{l} /$ well) were added to each reaction system for $6 \mathrm{~min}$. Immediately after staining, flow cytometric assays were conducted using a flow cytometer (EPICS; Beckman Coulter, Inc.). Analysis of flow cytometry results was conducted using FlowJo software7.6.1 (FlowJo, LLC).

Wound scratch assay. Cell migration was determined using a wound scratch assay. Briefly, $1 \times 10^{6}$ transfected cells/well were seeded into a 24 -well plate. When cells reached $100 \%$ confluence, a sterile $20-\mu 1$ pipette tip was used to produce a clear line in the wells. After washing with phosphate-buffered saline (PBS), the cells were grown in serum-free medium at $37^{\circ} \mathrm{C}$ for $24 \mathrm{~h}$. Under a light microscope, images of the cells were captured to record the wound width at $0 \mathrm{~h}$ and more images were captured after $24 \mathrm{~h}$. The migration distance was determined as the distance covered between 0 and $24 \mathrm{~h}$. 
Table I. Cox proportional hazards regression models for overall and recurrence-free survival among patients with colorectal cancer.

\begin{tabular}{|c|c|c|c|c|}
\hline \multirow[b]{2}{*}{ Parameter } & \multicolumn{2}{|c|}{ Univariate analysis } & \multicolumn{2}{|c|}{ Multivariate analysis } \\
\hline & $\mathrm{HR}(95 \% \mathrm{CI})$ & P-value & $\mathrm{HR}(95 \% \mathrm{CI})$ & P-value \\
\hline \multicolumn{5}{|l|}{ Disease-free survival } \\
\hline Location, rectum vs. colon & $1.98(0.85-4.60)$ & 0.112 & $1.67(0.71-3.90)$ & 0.237 \\
\hline T stage, T3-4 vs. T1-2 & $2.13(1.05-4.30)$ & 0.035 & $1.66(1.02-2.70)$ & 0.041 \\
\hline Node involvement, yes vs. no & $2.35(1.07-5.13)$ & 0.033 & $1.27(1.00-1.61)$ & 0.048 \\
\hline M stage, M1 vs. M0 & $2.56(1.23-5.33)$ & 0.012 & $1.51(1.04-2.20)$ & 0.031 \\
\hline Tumor differentiation, poor vs. well + moderate & $1.49(0.95-2.34)$ & 0.084 & $1.14(0.88-1.48)$ & 0.321 \\
\hline $\mathrm{CEA}, \geq 5$ vs. $<5 \mathrm{ng} / \mathrm{ml}$ & $1.40(0.68-2.86)$ & 0.362 & & \\
\hline Margin, positive vs. negative & $1.32(0.61-2.84)$ & 0.483 & & \\
\hline Chemotherapy, yes vs. no & $1.34(0.57-3.15)$ & 0.501 & & \\
\hline SNHG22, high vs. low & $2.47(1.13-5.41)$ & 0.023 & $1.68(1.04-2.71)$ & 0.034 \\
\hline \multicolumn{5}{|l|}{ Overall survival } \\
\hline Location, rectum vs. colon & $2.19(1.01-4.75)$ & 0.047 & $1.81(0.83-3.96)$ & 0.138 \\
\hline T stage, T3-4 vs. T1-2 & $1.59(1.00-2.53)$ & 0.050 & $1.33(0.97-1.82)$ & 0.077 \\
\hline Node involvement, yes vs. no & $1.91(1.12-3.26)$ & 0.018 & $1.57(1.06-2.33)$ & 0.025 \\
\hline M stage, M1 vs. M0 & $1.98(1.24-3.15)$ & 0.004 & $1.49(1.08-2.05)$ & 0.014 \\
\hline Tumor differentiation, poor vs. well + moderate & $1.39(0.66-2.93)$ & 0.395 & & \\
\hline $\mathrm{CEA}, \geq 5$ vs. $<5 \mathrm{ng} / \mathrm{ml}$ & $1.06(0.54-2.09)$ & 0.868 & & \\
\hline Margin, positive vs. negative & $0.97(0.45-2.08)$ & 0.930 & & \\
\hline SNHG22, high vs. low & $2.21(1.08-4.55)$ & 0.031 & $1.45(1.02-2.06)$ & 0.039 \\
\hline
\end{tabular}

All patients were divided into high or low SNHG22 expression groups using the median value as a cutoff point. Tumor classification and stage were determined using the 7th edition of UICC (2009) on cancer staging system. CEA, carcinoembryonic antigen; SHNG22, small nucleolar RNA host gene 22 .

Cell migration and invasion assay. Cell migration and invasion were examined using Transwell inserts (pore size, $8.0 \mu \mathrm{m}$ ) pre-coated without (for migration) or with (for invasion) Matrigel (on ice for $1 \mathrm{~h}$; BD Biosciences). Briefly, 2x10 $/$ well transfected cells in $200 \mu \mathrm{l}$ serum-free medium were suspended in the upper chamber, and the lower chamber was filled with $700 \mu \mathrm{l}$ medium containing $15 \% \mathrm{FBS}$. After $24 \mathrm{~h}$ at $37^{\circ} \mathrm{C}$ in an atmosphere containing $5 \% \mathrm{CO}_{2}$, the membranes in the lower chamber were fixed in $4 \%$ paraformaldehyde at room temperature for $24 \mathrm{~h}$ and stained with crystal violet for $18 \mathrm{~min}$. After washing with PBS, images of the cells on the membrane were captured under a light microscope in five randomly selected fields per sample.

Reverse transcription-quantitative PCR (RT-qPCR). Total RNA was extracted from frozen tissues and exponentially growing cells using TRIzol ${ }^{\circledR}$ (Invitrogen; Thermo Fisher Scientific, Inc.). Nuclear and cytoplasmic RNA were separated using the PARIS Kit (Thermo Fisher Scientific, Inc.). cDNA was synthesized from total RNA $(1,000 \mathrm{ng})$ using a reverse transcriptase cDNA synthesis kit (Takara Biotechnology Co., Ltd.) according to manufacturer's protocol. mRNA expression levels were measured using SYBR Premix Ex Taq II (Takara Biotechnology Co., Ltd.) on the CFX96 sequence detection system (Bio-Rad Laboratories, Inc.). The PCR cycling conditions were as follows: $95^{\circ} \mathrm{C}$ for $30 \mathrm{sec}$, followed by 40 cycles at $95^{\circ} \mathrm{C}$ for $5 \mathrm{sec}, 60^{\circ} \mathrm{C}$ for $30 \mathrm{sec}$, dissociation at $95^{\circ} \mathrm{C}$ for $15 \mathrm{sec}$, $60^{\circ} \mathrm{C}$ for $1 \mathrm{~min}$ and $95^{\circ} \mathrm{C}$ for $15 \mathrm{sec}$. The primer sequences are shown in Table SII. The RT-qPCR data were calculated using the comparative threshold cycle $\left(2^{-\Delta \Delta \mathrm{Cq}}\right)$ method with $\beta$-actin and U6 as the internal controls (16).

Western blotting. Total proteins were extracted from cells and tissues using RIPA buffer (Thermo Fisher Scientific, Inc.). The concentration of each protein was determined using a BCA protein assay kit (Pierce; Thermo Fisher Scientific, Inc.). Subsequently, equal amounts of cell lysates $(20 \mu \mathrm{g})$ were separated by sodium dodecyl sulfate-polyacrylamide gel electrophoresis on $10 \%$ gels, and then transferred to polyvinylidene difluoride membranes (MilliporeSigma). The membranes were blocked with TBS-3\% Tween-20 containing 5\% skim milk at room temperature for $24 \mathrm{~h}$ and blotted with a primary antibody against E2F3 (cat. no. ab50917; 1:2,000; Abcam) at $4^{\circ} \mathrm{C}$ for $16 \mathrm{~h}$. Subsequently, membranes were incubated with horseradish peroxidase-conjugated secondary antibodies (1:10,000; cat. no. ab205718; Abcam) on room temperature for $2 \mathrm{~h}$. After washing, immunoreactive bands were detected using chemiluminescence (Pierce; Thermo Fisher Scientific, Inc.). $\beta$-actin (cat. no. ab8227; 1:10,000; Abcam) was used as the loading control. Protein expression levels were semi-quantified by normalization against $\beta$-actin using ImageJ (version 1.36; National Institutes of Health). 
A

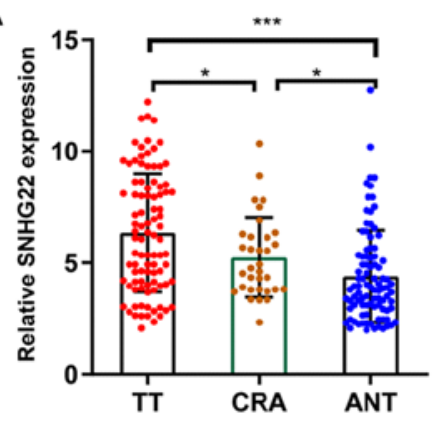

E

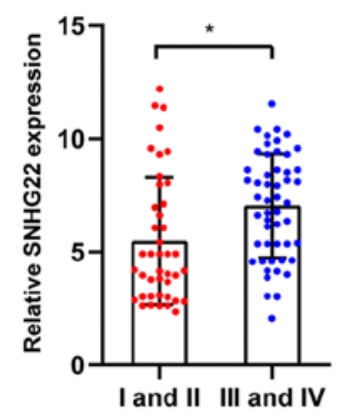

H

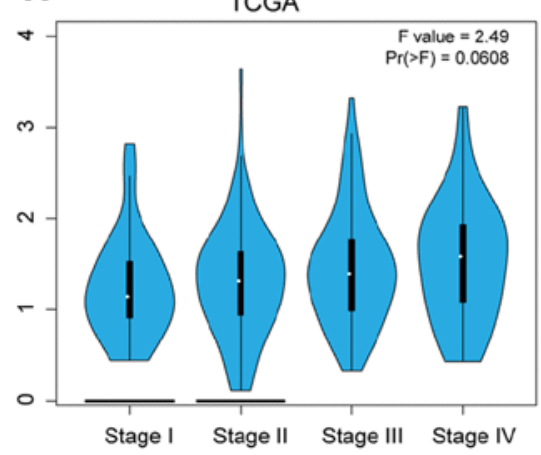

B

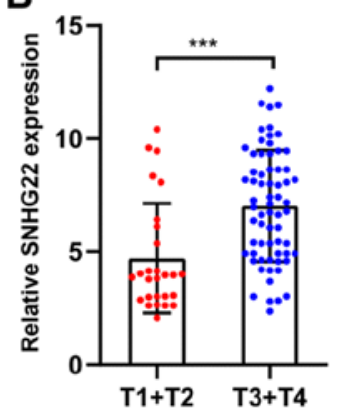

F

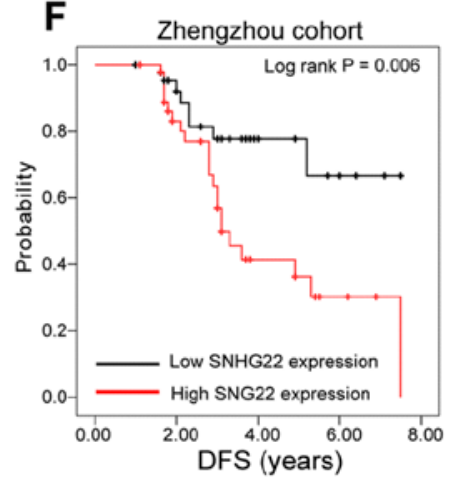

I

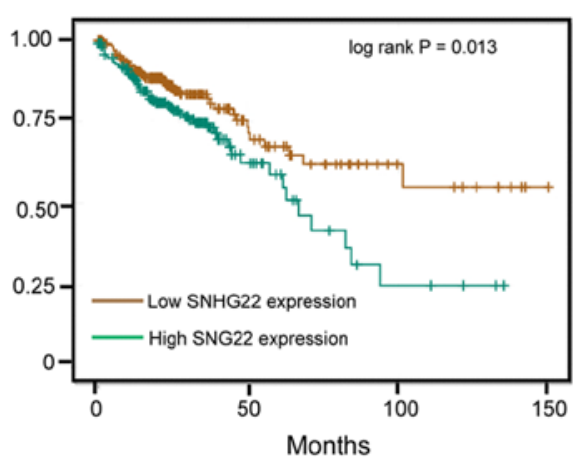

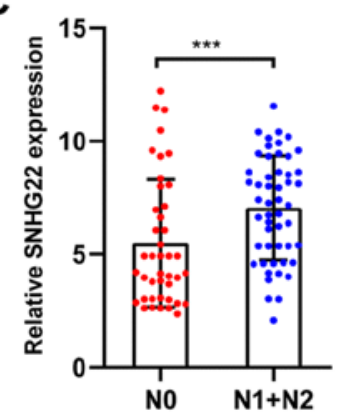

D

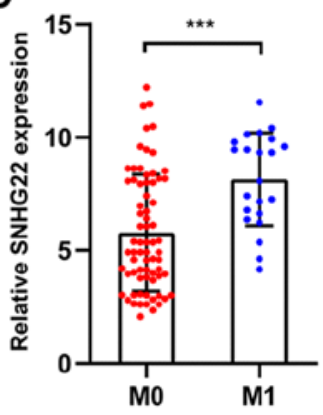

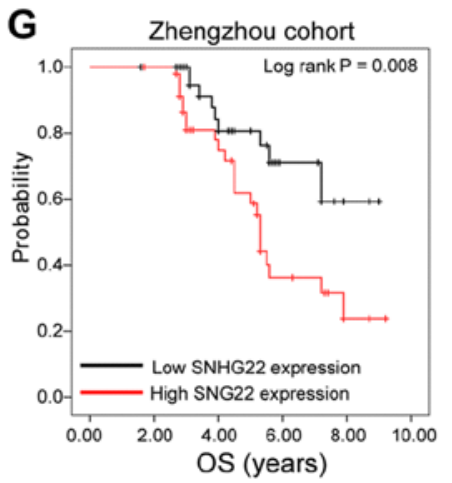

J

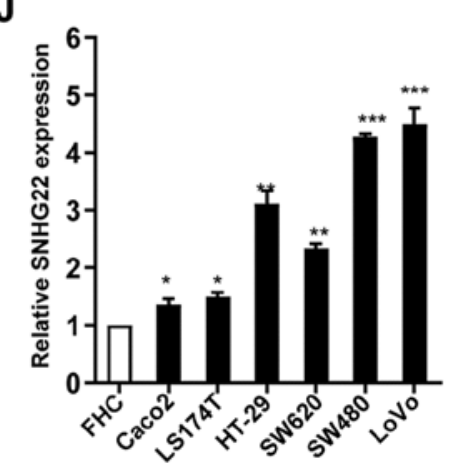

Figure 1. Differential expression of SNHG22 in CRC tissues and cell lines. (A) RT-qPCR analysis of the expression levels of SNHG22 in TTs (n=93), ANTs $(n=93)$ and CRA ( $n=33)$ colon tissues. Differential expression of SNHG22 in CRC tissues was analyzed according to (B) T stage, (C) node status, (D) distant metastasis and (E) TNM stage. Kaplan-Meier survival analysis of SNHG22 expression and (F) DFS and (G) OS in patients with CRC. (H) Expression of SNHG22 in patients with CRC split according to TNM stage in TCGA-COAD dataset. (I) OS in patients with CRC in TCGA-COAD dataset. (J) RT-qPCR analysis of the expression levels of SNHG22 in CRC cell lines and the FHC cell line, a human normal colon epithelial cell line. Data are presented as the mean \pm SD from triplicate experiments. ${ }^{*} \mathrm{P}<0.05,{ }^{* *} \mathrm{P}<0.01,{ }^{* * *} \mathrm{P}<0.001$ as indicated or vs. FHC. ANT, adjacent non-cancerous tissue; COAD, colorectal adenocarcinoma; CRA, colorectal adenoma; CRC, colorectal cancer; DFS, disease-free survival; OS, overall survival; RT-qPCR, reverse transcription-quantitative PCR; SNHG22, small nucleolar RNA host gene 22; TCGA, The Cancer Genome Atlas; TNM, tumor-node-metastasis; TT, tumor tissue.

Luciferase reporter assay. The predicted binding sites of miR-128-3p with the SNHG22 3' untranslated region (UTR) or E2F3 3'UTR were obtained from starBase v3.0 (http://starbase.sysu.edu.cn/). The mutant (mut) SNHG22 and E2F3 3' UTR luciferase reporter vectors were constructed using a Mutagenesis Kit (Qiagen, Inc.). The wild-type (wt) and mut sequences were each cloned into a psiCHECK 2 vector (Promega Corporation). CRC cells (1x10 $3 /$ well) and $293 \mathrm{~T}$ cells (1x10 $3 /$ well; American Type Culture Collection) in 96-well plates were co-transfected with wt/mut plasmid and miR-128-3p or NC $(50 \mu \mathrm{m})$ using Lipofectamine RNAiMAX (Invitrogen; Thermo Fisher Scientific, Inc.). After 36-h transfection at $4^{\circ} \mathrm{C}$, the cells were collected and tested using a dual-luciferase assay system (Promega Corporation) according to the manufacturer's instructions. pRL-TK was used as the internal control.
RNA immunoprecipitation (RIP). RIP was performed using an EZ-Magna RIP Kit (MilliporeSigma) and an anti-Argonaute-2 (Ago2) antibody (cat. no. ab32381; Abcam). CRC cells $\left(2 \times 10^{7}\right)$ were lysed in RIP buffer and centrifuged at 10,000 $\mathrm{x} \mathrm{g}$ at $4^{\circ} \mathrm{C}$ for $5 \mathrm{~min}$. The cell lysates were incubated with magnetic beads $(100 \mu \mathrm{l})$ conjugated with the anti-Ago 2 antibody (cat. no. ab32381; Abcam) or immunoglobulin G (cat. no. ab104155; Abcam) for $3 \mathrm{~h}$. After extensive washing using an elution buffer at $65^{\circ} \mathrm{C}$ for $10 \mathrm{~min}$, the purified RNA was analyzed by RT-qPCR.

Animal experiments. All animal experiments were approved by the Committee of the Ethics of Animal Experiments of Zhengzhou University (approval no. 20180376). A total of 15 male BALB/c nude mice (age, 4 weeks; weight, 14-16 g; Shanghai Experiment Animal Centre) were maintained under 
A

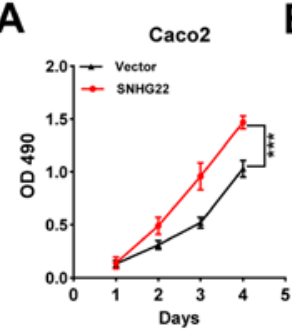

B

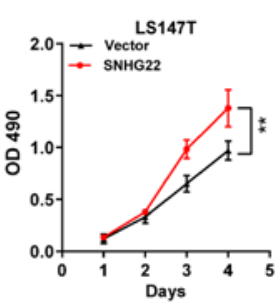

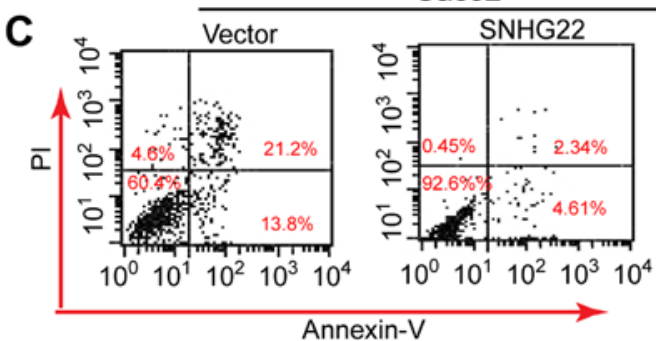

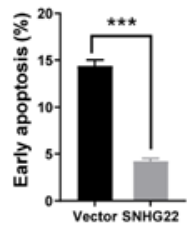

D

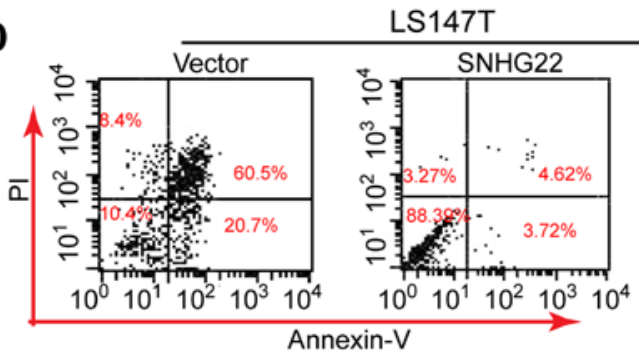

G

Caco2
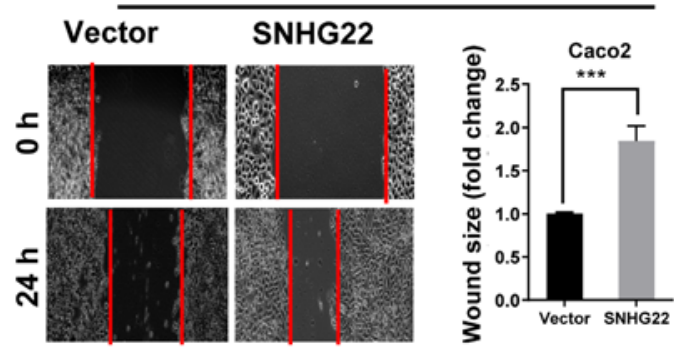

Caco2
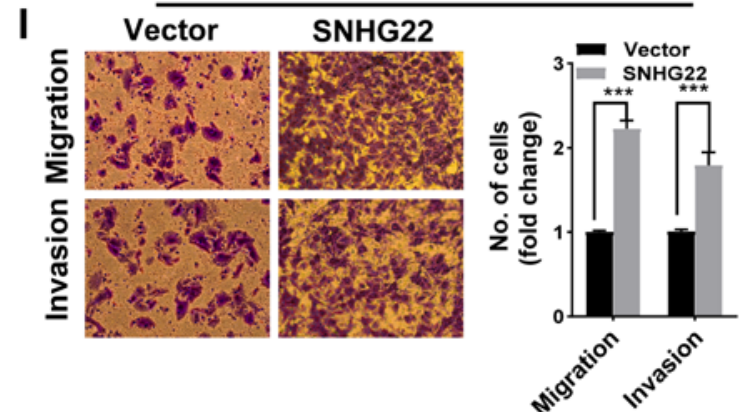
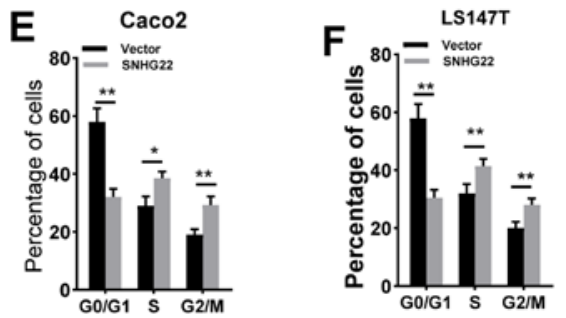

H

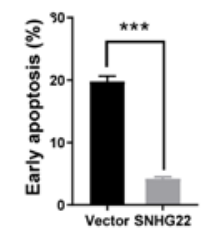

LS147T

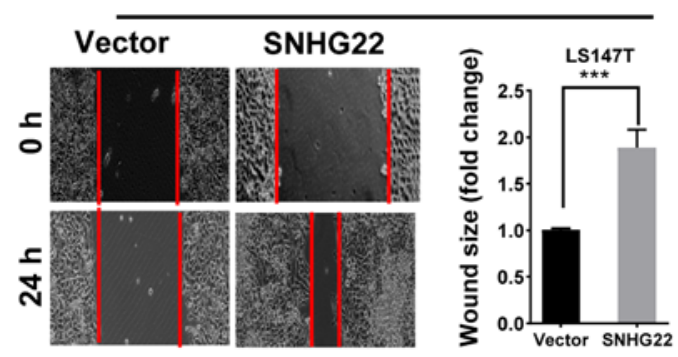

LS147T
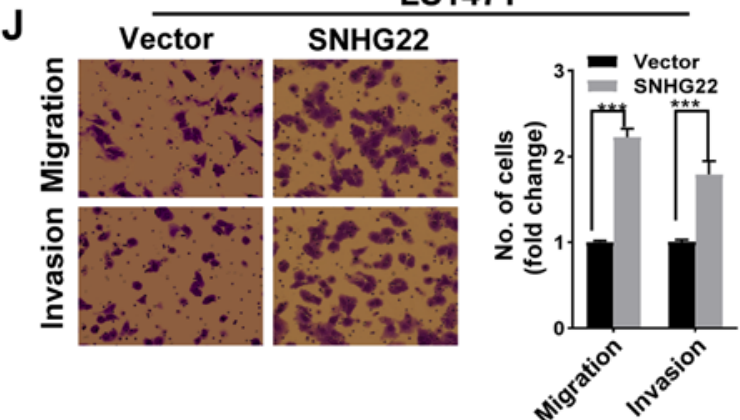

Figure 2. Overexpression of SNHG22 promotes colorectal cancer cell proliferation, apoptosis resistance, migration and invasion in vitro. Cell Counting Kit-8 assay analysis of the proliferative ability of (A) Caco2 and (B) LS147T cells transfected with the indicated vectors. Flow cytometric analysis of the $(\mathrm{C}$ and $\mathrm{D})$ apoptotic rates and (E and F) cell cycle progression of Caco2 and LS147T cells transfected with the indicated vectors. Wound scratch analysis of the migration of $(\mathrm{G})$ Caco2 and (H) LS147T cells transfected with the indicated vectors (magnification, $\mathrm{x} 200$ ). Transwell assay analysis of the migration and invasion of (I) Caco2 and (J) LS147T cells transfected with the indicated vectors (magnification, $\mathrm{x} 200$ ). Data are presented as the mean \pm SD from triplicate experiments. ${ }^{*} \mathrm{P}<0.05,{ }^{* *} \mathrm{P}<0.01,{ }^{* * *} \mathrm{P}<0.001$. NC, negative control; OD, optical density; PI, propidium iodide; SNHG22, small nucleolar RNA host gene 22.

specific pathogen-free conditions and randomly subdivided into three subgroups. LoVo cells that had been stably infected with $\mathrm{Lv}$-shNC, Lv-sh\#1 and Lv-sh\#2 vectors were harvested, and $6 \times 10^{6}$ cells were injected subcutaneously into the dorsal flank region of each mouse. Tumor growth was measured every 5 days, and was calculated using the following formula: Volume $=\left(\right.$ width $^{2} \mathrm{x}$ length $) / 2$. Euthanasia was performed by intraperitoneal injection of $200 \mathrm{mg} / \mathrm{kg}$ pentobarbital (17). Subsequently, the weight of each tumor was evaluated, and the tumor samples were processed for RT-qPCR, western blotting and immunohistochemistry (IHC). The present study was carried out according to the National Institutes of Health Guide for the Care and Use of Laboratory Animals (18).

Hematoxylin-eosin (HE) staining and IHC. The tumors from the nude mice were fixed in $10 \%$ paraformaldehyde at $4^{\circ} \mathrm{C}$ for $12 \mathrm{~h}$, and then dehydrated in different concentrations of ethanol, permeabilized using xylene and embedded in paraffin. The paraffin-embedded tumor tissues were then sliced $(0.5 \mu \mathrm{m})$, rehydrated, and were stained with $\mathrm{HE}$ at $4^{\circ} \mathrm{C}$ for $10 \mathrm{~min}$ and sealed with neutral gum. For IHC assessment of E2F3 and $\mathrm{Ki}-67$ in colorectal tumor tissues from the nude mice, the DAKO 


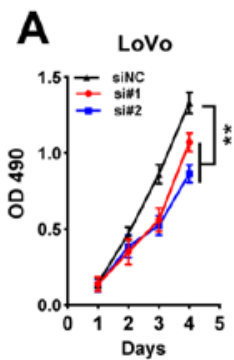

B

LoVo
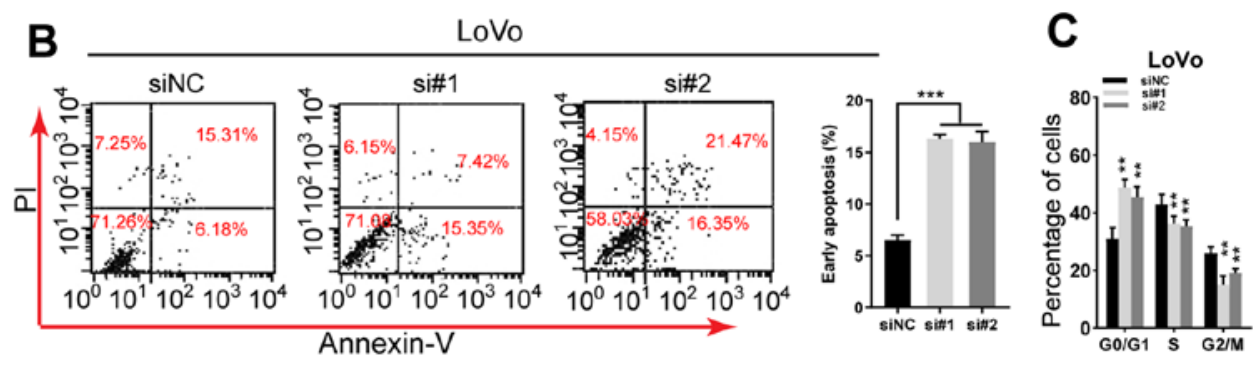

LoVo

D

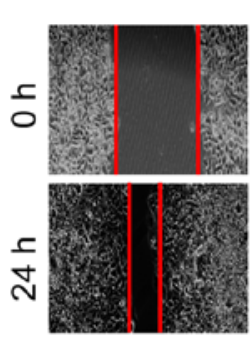

siNC

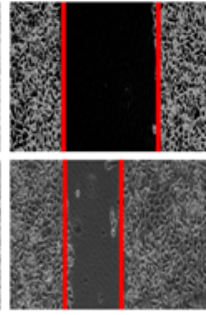

si\#1

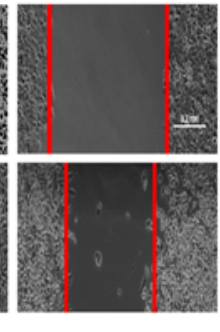

si\#2

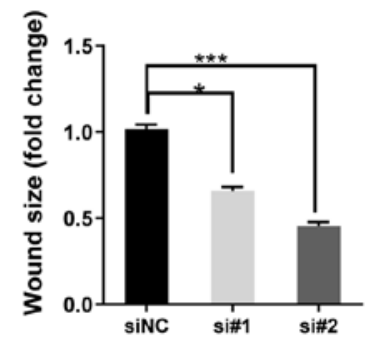

E

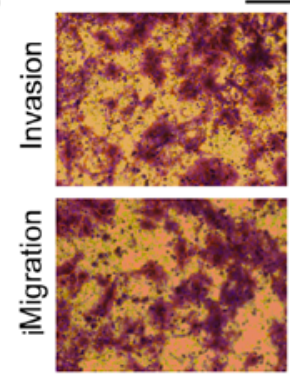

siNC

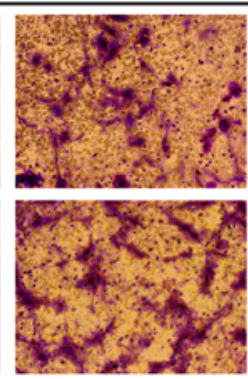

si\#1

LoVo

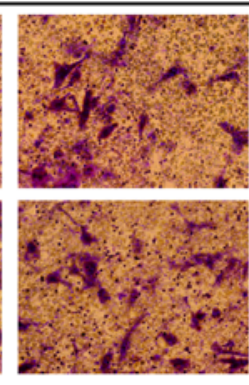

si\#2

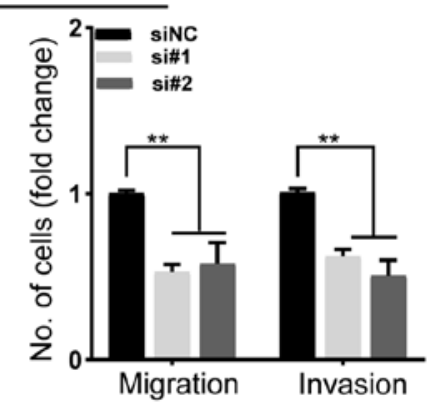

Figure 3. Knockdown of SNHG22 inhibits colorectal cancer cell proliferation, apoptosis resistance, migration and invasion in vitro. (A) Cell Counting Kit-8 assay analysis of the proliferative ability of LoVo cells after transfection with the indicated sequences. Flow cytometric analysis of the (B) apoptotic rates and (C) cell cycle progression of LoVo cells after transfection with the indicated sequences. (D) Wound scratch analysis of the migration of LoVo cells after transfection with the indicated sequences (magnification, x200). (E) Transwell invasion assay analysis of the invasion of LoVo cells after transfection with the indicated sequences (magnification, $\mathrm{x} 200$ ). Data are presented as the mean $\pm \mathrm{SD}$ from triplicate experiments. ${ }^{*} \mathrm{P}<0.05,{ }^{* *} \mathrm{P}<0.01,{ }^{* * *} \mathrm{P}<0.001$ as indicated or $\mathrm{vs}$. siNC. NC, negative control; OD, optical density; PI, propidium iodide; si, small interfering; SNHG22, small nucleolar RNA host gene 22.

Envision system (Dako; Agilent Technologies, Inc.) was used. Briefly, after paraffin-embedded sections of tumor tissues were heated at $60^{\circ} \mathrm{C}$, the sections were incubated with primary antibodies against E2F3 (1:200; cat. no. ab50917; Abcam) and Ki-67 (1:1,000; cat. no. ab279653; Abcam) overnight at $4^{\circ} \mathrm{C}$. Then, the sections were incubated with biotin-labeled secondary antibodies (1:1,000; cat. no. ab205718; Abcam) at $37^{\circ} \mathrm{C}$ for $20 \mathrm{~min}$. Under a light microscope, IHC staining scores for $\mathrm{E} 2 \mathrm{~F} 3$ were obtained by multiplying the intensity $(0$, negative; 1 , low; 2 , medium; and 3 , high) with the extent of staining $(0,0 \% ; 1,0-10 \% ; 2,10-50 \% ; 3$, $50-75 \% ; 4,>75 \%$ ); the final scores were between $0-12$. For evaluation of Ki67, the number of positive cells was calculated in three representative areas of high staining.

Bioinformatics and statistical analyses. IncLocator (19) was used to analyze lncRNA subcellular locations. CPAT (http://lilab.research.bcm.edu/cpat/index.php) and LNCipedia (https://lncipedia.org) were used to analyze the protein coding probability. The online tools TargetScan (http://www. targetscan.org/), miRwalk (http://mirwalk.umm. uni-heidelberg.de), miRTarbase (http://mirtarbase.mbc.nctu. edu.tw/index.html), miRDB (http://www.mirdb.org/), and starBase (http://starbase.sysu.edu.cn/index.php) were used to analyze potential target genes of miR-128-3p. Analysis of data from The Cancer Genome Atlas (TCGA)-colon adenocarcinoma (COAD) $(n=267)$ was conducted using starBase and UALCAN (http://ualcan.path.uab.edu/index.html) online tools. All data are presented as the mean \pm standard deviation from triplicate experiments. Regarding comparisons, Student's t-test (two groups) or one-way ANOVA with Scheffe test (multiple groups) was conducted as appropriate. To compare SNHG22 expression levels in TT, ANT and CRA samples, paired samples were analyzed using a paired Student's t-test and independent samples were analyzed using unpaired Student's t-tests, after which, a Bonferroni correction was applied. To compare E2F3 and Ki-67 staining scores in mice tumor samples, Kruskal-Wallis and Dunn's post hoc test was used. Correlations were calculated using Spearman's correlation analysis. The survival curves were constructed using the Kaplan-Meier method and were analyzed using the log-rank 
A

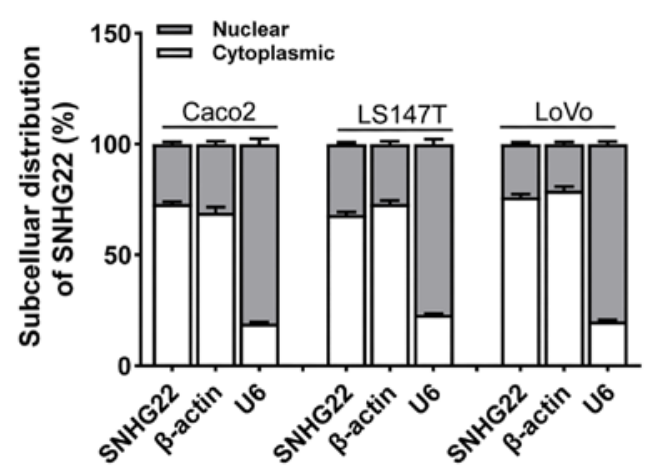

B

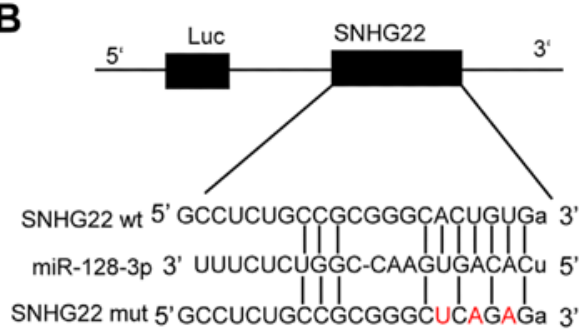

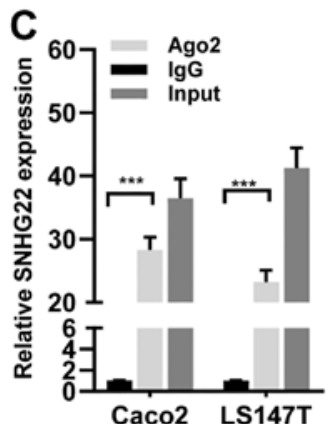

D

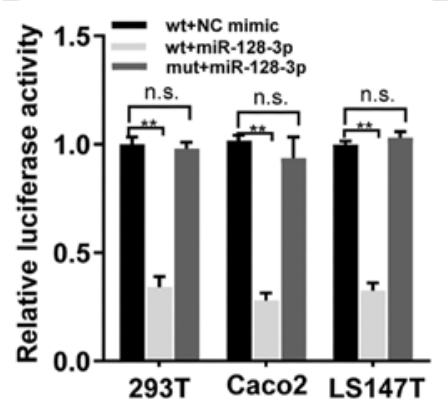

E

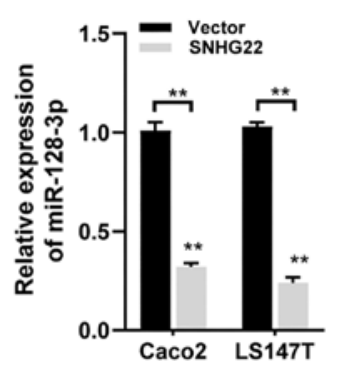

F

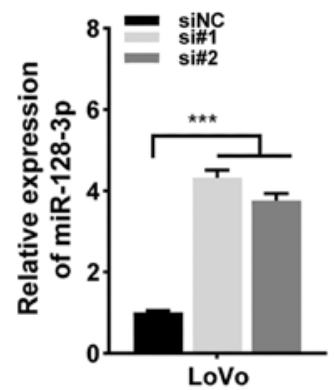

G

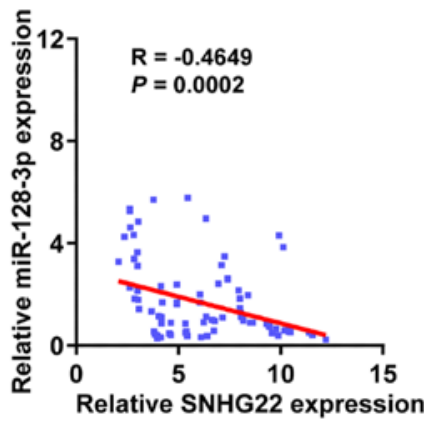

Figure 4. SNHG22 functions as a competing endogenous RNA to regulate miR-128-3p expression in CRC. (A) Subcellular localization of SNHG22 in CRC cell lines (Caco2, LS147T and LoVo). $\beta$-Actin and U6 served as cytoplasmic and nuclear localization markers, respectively. (B) Predicted binding sites of miR-128-3p to the SNHG22 sequence. (C) RNA immunoprecipitation assay was performed with an antibody against Ago2 or IgG in Caco2 and LS147T cell lines. (D) Luciferase activity of $293 \mathrm{~T}$, Caco2 and LS147T cell lines co-transfected with miR-128-3p mimic (or NC mimic) and luciferase reporters containing SNHG22 wt or SNHG22 mut transcript was analyzed. (E and F) Reverse transcription-quantitative PCR analysis of the expression levels of miR-128-3p in CRC cells after transfection with the indicated vectors and sequences. (G) Negative correlation between SNHG22 and miR-128-3p expression in human CRC tissues from the Zhengzhou cohort (Spearman's correlation analysis; $\mathrm{R}=-0.4649, \mathrm{P}=0.002$ ). Data are presented as the mean $\pm \mathrm{SD}$ from triplicate experiments. ${ }^{* *} \mathrm{P}<0.01,{ }^{* * *} \mathrm{P}<0.001$. Ago2, Argonaute2; CRC, colorectal cancer; EV, empty vector; miR, microRNA; mut, mutant; NC, negative control; n.s., not significant; si, small interfering; SNHG22, small nucleolar RNA host gene 22; wt, wild-type.

test. Crude and adjusted hazard ratios (HRs) and $95 \%$ confidence intervals (CIs) were calculated using Cox proportional hazards modeling. Potential confounders were included in the multivariate analysis at a significance level of $\mathrm{P}<0.15$, as determined by univariate analysis. All statistical analyses were conducted using the PASW Statistics 19.0 software program (SPSS, Inc.) and GraphPad Prism version 8.0 (GraphPad, Inc.). $\mathrm{P}<0.05$ was considered to indicate a statistically significant difference.

\section{Results}

SNHG22 is upregulated in CRC tissues and cell lines, and is associated with poor prognosis in patients with CRC. To investigate SNHG22 expression levels in CRC, RT-qPCR analysis was performed to examine SNHG22 levels in TT and ANT colorectal samples ( $n=93$ pairs) and human CRA samples ( $n=32)$. SNHG22 expression was significantly higher in TTs compared with that in CRA tissues and ANTs (Fig. 1A). Additionally, high SNHG22 expression was significantly associated with advanced T stage (Fig. 1B), lymph node metastasis (Fig. 1C), distant metastasis (Fig. 1D), advanced clinical stage (Fig. 1E), and poor disease-free survival (DFS) and overall survival (OS) (Fig. 1F and G). Data from TCGA confirmed these results (Fig. 1H and I). Multivariate Cox regression analysis validated high SNHG22 expression as a predictor of poor DFS (HR, 1.68; 95\% CI, 1.04-2.71) and OS (HR, 1.45; 95\% CI, 1.02-2.06; Table I). High SNHG22 expression was also confirmed in the CRC cell lines (LS174T, LoVo, Caco2, SW480, HT-29 and SW620) compared with that in the FHC cell line (Fig. 1J). These results indicated that SNHG22 was highly expressed in CRC tissues and cell lines, and may be associated with poor survival in CRC. Furthermore, the coding potential of SNHG22 was calculated using CPAT and LNCipedia; the results revealed that SNHG22 had very low coding probability, thus indicating that it may be a ncRNA (Fig. S1).

Overexpression of SNHG22 stimulates CRC cell proliferation, apoptosis resistance, migration, and invasion in vitro. To investigate the role of SNHG22 in the progression of CRC cells, gain-of-function experiments were performed using the Caco2 and LS174T cell lines because both cell lines had low SNHG22 expression levels. SNHG22 expression was significantly enhanced after the cells had been transfected with the SNHG22 vector (Fig. S2A). Functionally, upregulating SNHG22 expression promoted the proliferation, $\mathrm{G}_{2} / \mathrm{M}$ phase arrest and apoptosis resistance of both cell lines (Figs. 2A-F and S2B). Furthermore, the wound scratch and Transwell assays demonstrated a marked enhancement in the migratory and invasive ability of SNHG22-overexpressing cells (Fig. 2G-J). These data indicated that SNHG22 overexpression may promote CRC cell proliferation, apoptosis resistance, migration and invasion. 
A

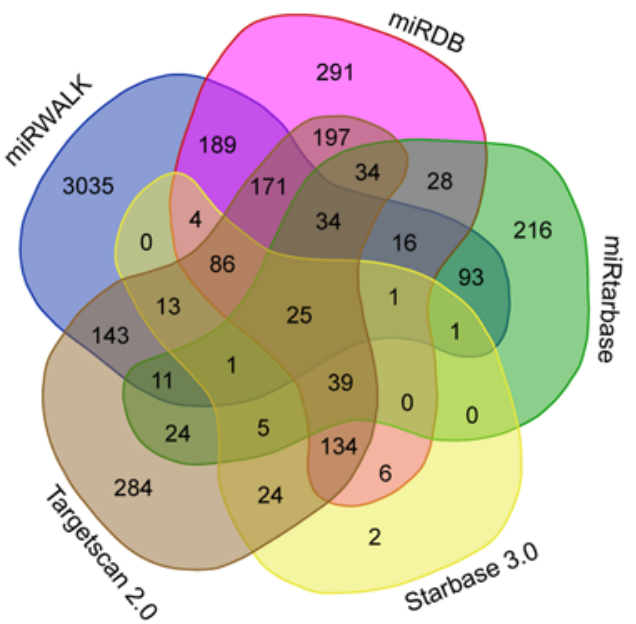

B

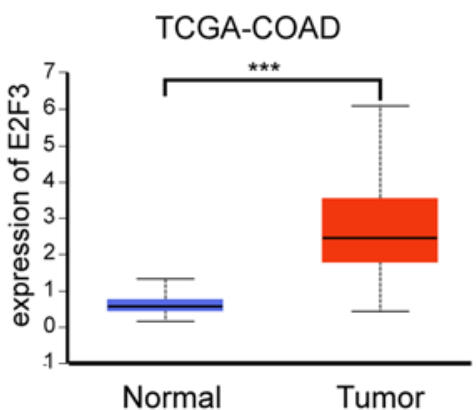

C

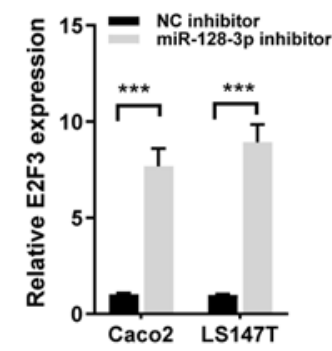

D

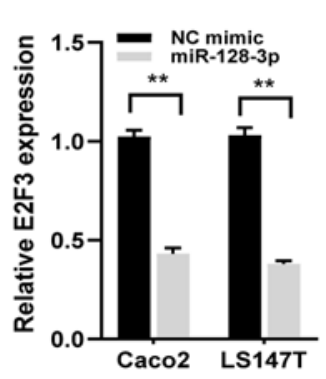

$\mathbf{E}$

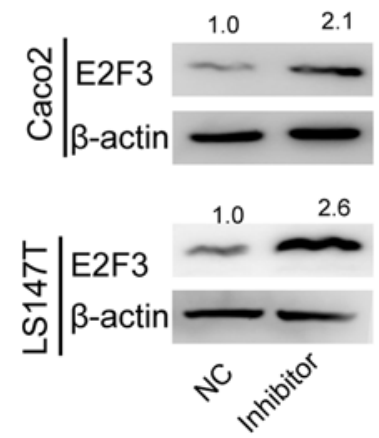

$\mathbf{F}$

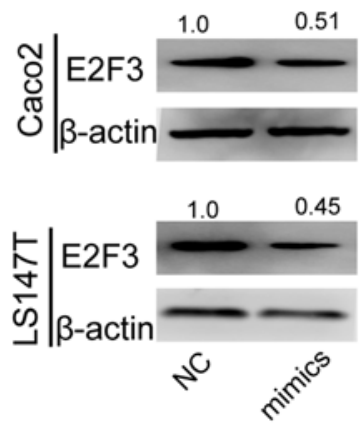

G

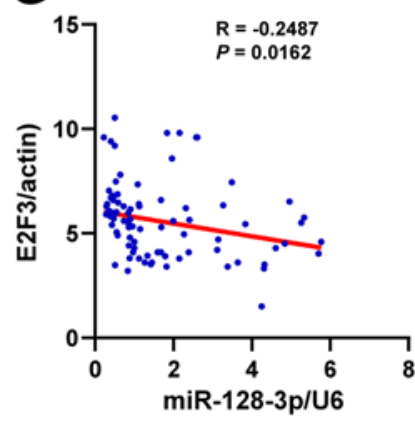

\section{H}

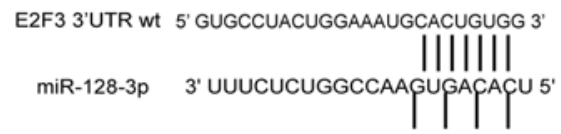

E2F3 3'UTR mut 5' GUGCCUACUGGAAAUGCGCGGGGG 3'

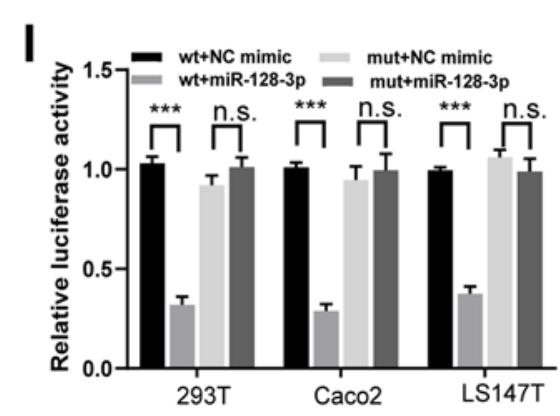

Figure 5. miR-128-3p targets and regulates E2F3. (A) A Venn diagram showing the number of genes identified as potential targets of miR-128-3p. (B) Expression levels of E2F3 in tumor and unmatched normal tissues in TCGA-COAD dataset. (C and D) Reverse transcription-quantitative PCR and (E and F) western blot analysis of the mRNA and protein expression levels of E2F3 in Caco2 and LS147T cells after transfection with the indicated sequences. Protein expression levels were semi-quantified by normalization against $\beta$-actin with ImageJ. (G) A significant inverse association was detected between miR-128-3p and E2F3 mRNA expression in human CRC tissues from the Zhengzhou cohort $(\mathrm{R}=-0.2487, \mathrm{P}=0.016)$. $(\mathrm{H}) \mathrm{miR}-128-3 \mathrm{p}$ putative binding sites and corresponding mut sequence of E2F3. (I) Dual-luciferase reporter assays in CRC cell lines co-transfected with miR-128-3p mimic and luciferase reporters containing wt or mut E2F3 transcripts. The relative luciferase activity was normalized to the Renilla luciferase activity. (J) A positive correlation between SNHG22 and E2F3 expression in human $\mathrm{CRC}$ tissues from Zhengzhou cohort $(\mathrm{R}=-0.4047, \mathrm{P}<0.001)$. Data are presented as the mean $\pm \mathrm{SD}$ from triplicate experiments. ${ }^{* *} \mathrm{P}<0.01$, ${ }^{* * *} \mathrm{P}<0.001$. COAD, colon adenocarcinoma; CRC, colorectal cancer; E2F3, E2F transcription factor 3; miR, microRNA; mut, mutant; NC, negative control; n.s., not significant; TCGA, The Cancer Genome Atlas; UTR, untranslated region; wt, wild-type.

Knockdown of SNHG22 inhibits CRC cell proliferation, apoptosis resistance, migration, and invasion in vitro. To determine whether silencing SNHG22 would affect CRC cell biological function, two parallel siRNAs targeting SNHG22 (i.e., si\#1 and si\#2) were used for the knockdown experiments. RT-qPCR confirmed the knockdown efficiency of both siRNAs in the LoVo cell line (Fig. S3A). The results of CCK-8 assay and flow cytometry demonstrated a marked decrease in LoVo cell proliferation, $\mathrm{G}_{2} / \mathrm{M}$ phase arrest and apoptosis resistance in cells with SNHG22 knockdown (Figs. 3A-C and S3B). Furthermore, the wound scratch and Transwell assays indicating that silencing SNHG22 attenuated LoVo cell migratory and invasive ability (Fig. 3D and E). These data indicated that knocking down SNHG22 may inhibit CRC cell proliferation, migration and invasion.

SNHG22 functions as a competing endogenous RNA (ceRNA) to regulate miR-128-3p expression in CRC cells. As the 
A

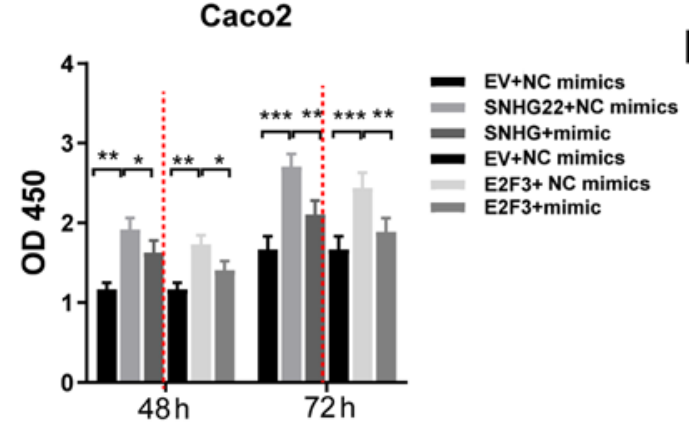

B

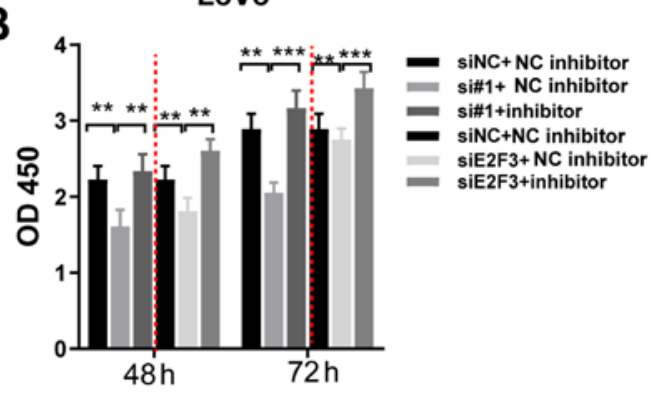

C

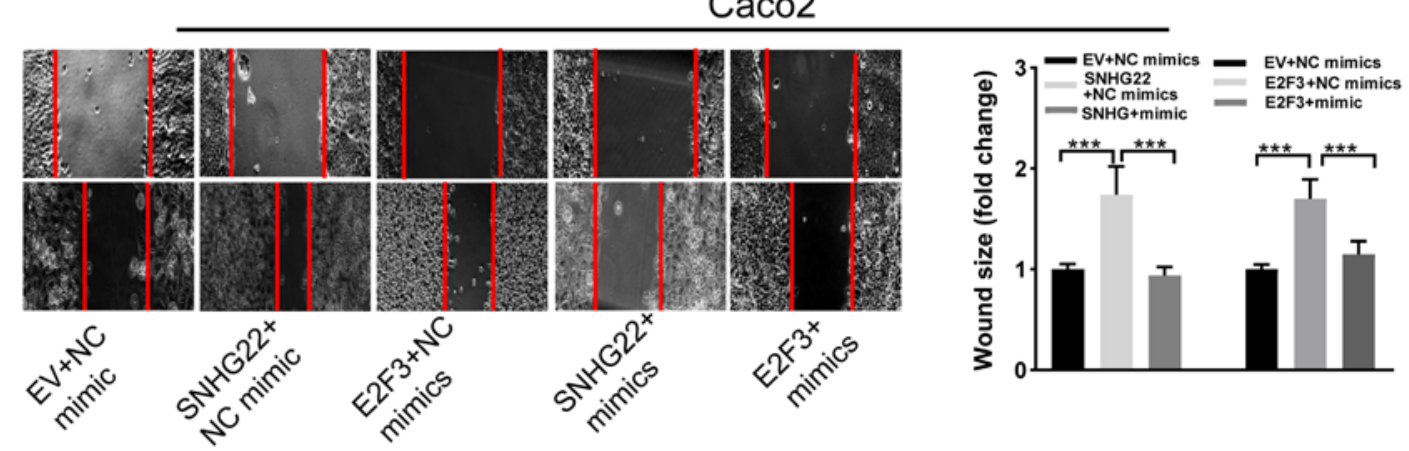

D

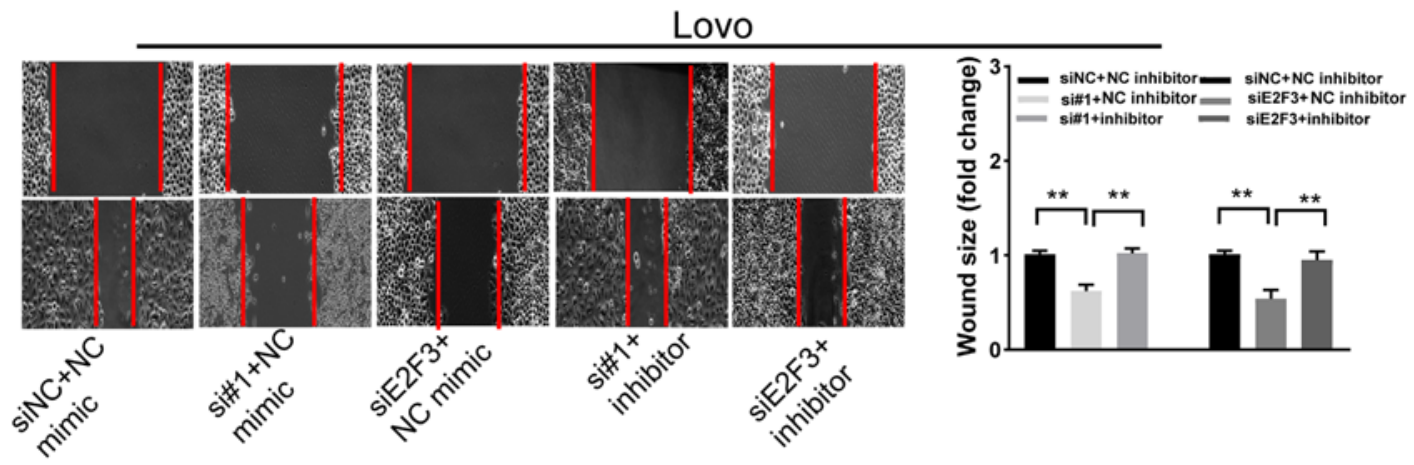

E

Caco2

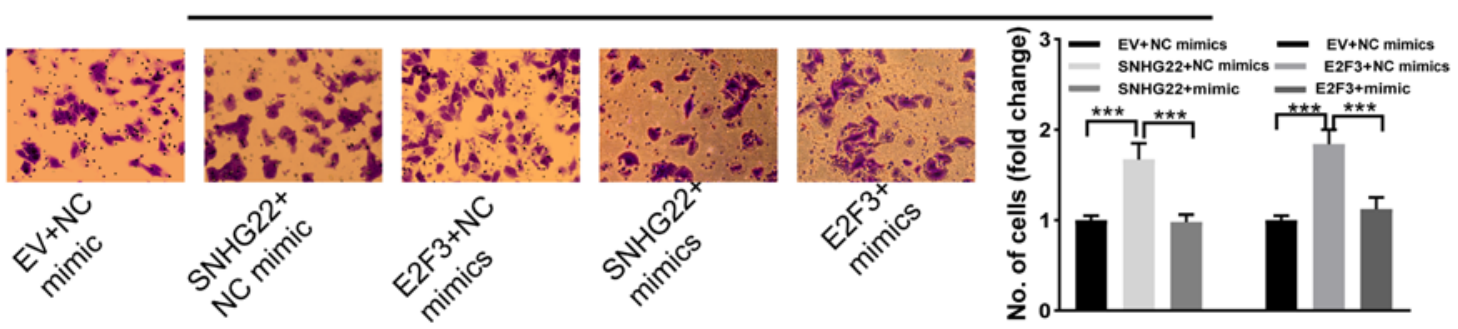

F

Lovo
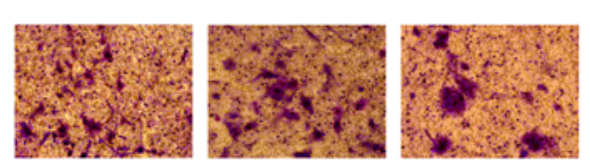

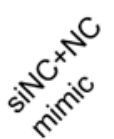
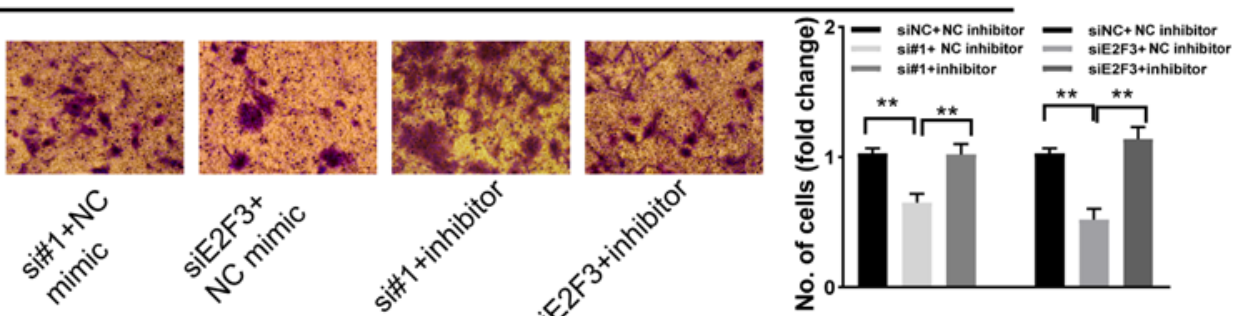

Figure 6. SNHG22 exerts its function by inhibiting the miR-128-3p/E2F3 axis in CRC cells. Cell Counting Kit-8 assay analysis of the proliferative ability of (A) $\mathrm{Caco} 2$ and (B) LoVo cells after transfection with the indicated vectors and sequences. Wound scratch analysis of the migratory ability of (C) Caco2 and (D) LoVo cells after transfection with the indicated vectors and sequences (magnification, $\mathrm{x} 200$ ). Transwell assay analysis of the invasive ability of (E) Caco2 and (F) LoVo cells after transfection with the indicated vectors and sequences (magnification, x200). Data are presented as the mean \pm SD from triplicate experiments. ${ }^{*} \mathrm{P}<0.05,{ }^{* * *} \mathrm{P}<0.01,{ }^{* * *} \mathrm{P}<0.001$. E2F3, E2F transcription factor 3; EV, empty vector; miR, microRNA; NC, negative control; OD, optical density; si, small interfering; SNHG22, small nucleolar RNA host gene 22. 
A

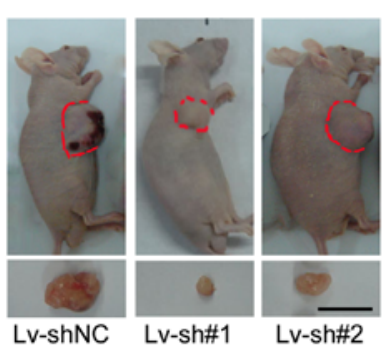

B

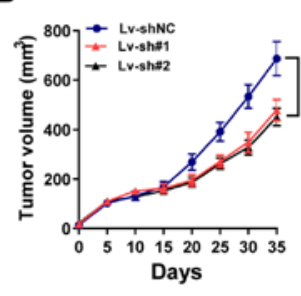

C

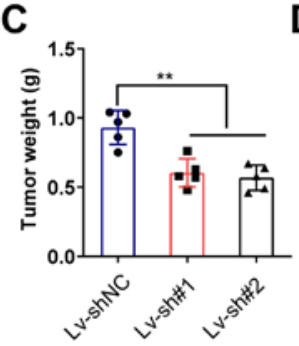

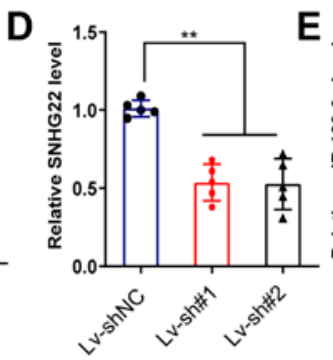

E

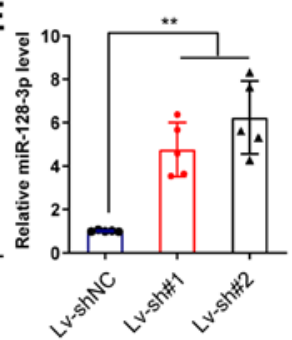

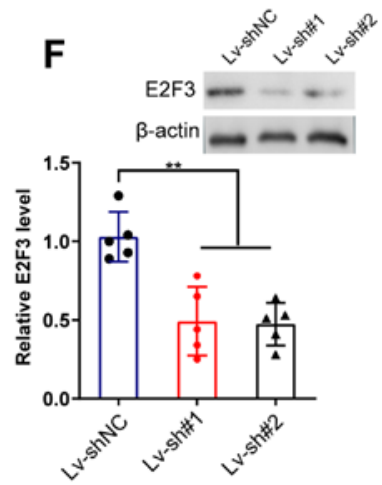

G
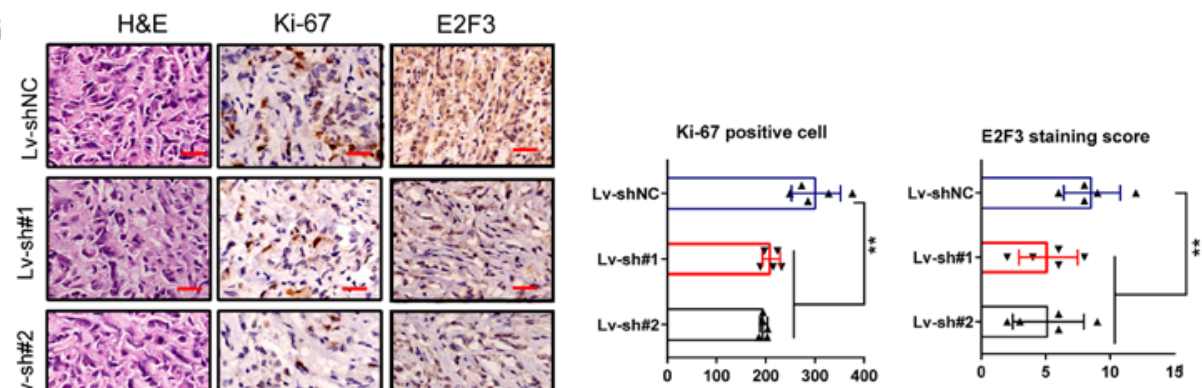

Figure 7. Knockdown of SNHG22 inhibits tumor xenograft growth of CRC in vivo. (A) LoVo cells were inoculated in BALB/c nude mice ( $\mathrm{n}=5 / \mathrm{group}$ ) to establish subcutaneous xenograft tumors, and images of the dissected tumors were captured. Scale bar, $10 \mathrm{~mm}$. Effects of SNHG22 knockdown in LoVo cells on (B) tumor volume and (C) tumor weight in the subcutaneous xenograft mouse models. (D and E) Reverse transcription-quantitative PCR and (F) western blot analysis of the expression levels of SNHG22, miR-128-3p and E2F3 in the subcutaneous xenograft mouse models. (G) Immunohistochemical analysis of the expression levels of Ki-67 and E2F3 in the subcutaneous xenograft mouse models. Scale bar, $100 \mu \mathrm{m}$. Data are presented as the mean \pm SD from triplicate experiments. ${ }^{* *} \mathrm{P}<0.01,{ }^{* * *} \mathrm{P}<0.001$. E2F3, E2F transcription factor 3; HE, hematoxylin and eosin; Lv, lentivirus; miR, microRNA; NC, negative control; sh, short hairpin; SNHG22, small nucleolar RNA host gene 22.

subcellular localization of lncRNAs can indicate their function, the results from IncLocator (19) demonstrated that SNHG22 was preferentially localized in the cytoplasm (Fig. S4). Subsequent cellular fractionation experiments confirmed this in CRC cells (Caco2, LS174T and LoVo; Fig. 4A). The starBase v3.0 findings indicated that SNHG22 contains complementary binding sites to miR-128-3p seed regions (Fig. 4B). To test this hypothesis, RIP experiments were performed with anti-Ago 2 in Caco2 and LS174T cells, and observed enrichment of SNHG22 with the Ago2 antibody was detected (Fig. 4C), indicating that SNHG22 had complementary binding sites to miR-128-3p and directly bound to miR-128-3p. To explore whether SNHG22 is regulated by miR-128-3p, dual-luciferase reporter plasmids were constructed containing wt or mut putative binding sites of SNHG22 transcripts. Luciferase activity was markedly reduced in $293 \mathrm{~T}$ cells that had been co-transfected with SNHG22 wt and miR-128-3p mimic compared with those transfected with SNHG22 wt and NC mimic. However, no statistical changes in luciferase activity were observed in cells transfected with the mut reporter. These results were confirmed in the Caco2 and LS174T cell lines (Fig. 4D). Furthermore, RT-qPCR revealed that SNHG22 overexpression significantly decreased miR-128-3p expression levels in CRC cells, whereas silencing SNHG22 enhanced miR-128-3p expression (Fig. 4E and F). SNHG22 expression had a significant inverse relationship with miR-128-3p expression in human CRC tissues from the Zhengzhou cohort (Fig. 4G). These findings demonstrated that SNHG22 may act as a miR-128-3p sponge in CRC.

miR-128-3p targets and regulates E2F3. Five independent algorithmic programs were used to define putative targets of miR-128-3p, and identified 25 common predicted targets (Fig. 5A). Among these, E2F3 was selected because TCGA-COAD data indicated that E2F3 can enhance CRC progression and may be associated with tumor (Figs. 5B, and S5A and B). After successfully transfecting miR-128-3p mimics and inhibitor into CRC cells (Fig. S5C and D), it was revealed that miR-128-3p overexpression markedly decreased the mRNA and protein expression levels of E2F3 in Caco2 and LS174T cells, whereas silencing miR-128-3p enhanced the expression levels of E2F3 (Fig. 5C-F). Moreover, Spearman's correlation analysis identified a significant inverse relationship between the expression levels of E2F3 mRNA and miR-128-3p in human CRC tissues from the Zhengzhou cohort (Fig. 5G). To determine if E2F3 is an authentic target of miR-128-3p, luciferase reporter gene constructs were generated wherein the E2F3 3'UTR sequences were fused with the Renilla luciferase coding sequence (Fig. 5H). The upregulation of miR-128-3p significantly decreased the luciferase activity of the E2F3 wt 3'UTR. (Fig. 5I). Nevertheless, altering miR-128-3p expression had no significant effects on the mut construct (Fig. 5I). In addition, E2F3 mRNA expression had a meaningful positive relationship with $\mathrm{SNHG} 22$ in human CRC tissues from the Zhengzhou cohort (Fig. 5J). These data suggested that E2F3 could be a direct target of miR-128-3p in CRC cells.

SNHG22 exerts its function by inhibiting the miR-128-3p/E2F3 axis in CRC cells. Functional rescue experiments were performed to examine whether the miR-128-3p/E2F3 axis mediates the biological roles of SNHG22 in CRC cells. The results of RT-qPCR analyses confirmed the transfection efficiencies of E2F3 overexpression and siRNA vectors in 
CRC cells (Fig. S6A and B). SNHG22 or E2F3 overexpression stimulated $\mathrm{Caco} 2$ cell proliferation, apoptosis resistance, migration and invasion, whereas upregulating miR-128-3p reversed these effects. By contrast, knocking down SNHG22 or E2F3 significantly weakened the LoVo cell malignant phenotype; nevertheless, these inhibitory effects were attenuated by co-transfection with miR-128-3p inhibitors (Figs. 6A-F, and $\mathrm{S} 6 \mathrm{C}$ and D). These results indicated that the miR-128-3p/E2F3 axis reversed the stimulatory effects of $S N H G 22$ on the proliferative, migratory and invasive capacity of CRC cells.

Knockdown of SNHG22 inhibits CRC tumor xenograft growth in vivo. A tumor xenograft assay was performed to confirm the roles of SNHG22 in CRC growth in vivo. As shown in Fig. 7A-C, compared with in the $\mathrm{Lv}$-shNC group, the tumor size, volume and weight in mice in the $\mathrm{Lv}$-sh\#1 and Lv-sh\#2 groups were markedly reduced $(\mathrm{P}<0.01)$. Furthermore, $\mathrm{RT}-\mathrm{qPCR}$ verified the decreased expression levels of SNHG22 and E2F3, and the increased expression levels of miR-128-3p in tumors from the $\mathrm{Lv}$-sh\#1 and Lv-sh\#2 groups compared with those in the shNC group (Fig. 7D-F). Immunohistochemistry showed that tumors from the Lv-sh\#1 and Lv-sh\#2 groups had decreased expression levels of Ki-67 and E2F3 staining compared with those in tumors from the shNC group (Fig. 7G).

\section{Discussion}

Accumulating evidence has recently revealed that the dysregulation of lncRNAs serves an essential role in the development and invasion of human cancer, including CRC $(20,21)$. The present study revealed that $S N H G 22$ expression was significantly elevated in CRC tissues and cell lines. High levels of SNHG22 expression were significantly associated with unfavorable clinicopathological characteristics and worse survival in patients with CRC. Functionally, ectopic SNHG22 overexpression drove proliferation, apoptosis resistance, migration and invasion in CRC cell lines. Knocking down SNHG22 inhibited xenograft tumor growth in vivo. The present study confirmed that $S N H G 22$ performed its tumor-promoting function by sponging miR-128-3p, and enhancing the expression and activity of E2F3. To the best of our knowledge, the present results are the first to clarify that $S N H G 22$ acts as an oncogene in CRC, and that it may be used as a potential therapeutic target for this disease.

The current anatomically based TNM staging system cannot precisely distinguish the risk of recurrence/distant metastasis in patients with CRC; therefore, it is essential to identify novel prognostic biomarkers. The dysregulation of lncRNAs in various human tumors is associated with excellent or poor survival, thus making them promising prognostic biomarkers $(22,23)$. For example, $S N H G 7$ expression has been reported to be enhanced in CRC tissues compared with that in non-cancerous tissues, and this high expression was related to aggressive pathological characteristics, such as TNM stage, lymphatic metastasis and distant metastasis, as well as poor prognosis $(9,10)$. The upregulation of Pvt1 oncogene has also been reported to indicate poor survival in several types of cancer, including CRC (23). Some researchers have reported upregulated $S N H G 22$ expression in EOC, PTC and clear cell renal cell carcinoma, and high
SNHG22 expression may indicate poor prognosis in these three human malignancies $(12,14,24)$. The present study first explored the expression patterns of $S N H G 22$ in CRC TTs and ANTs, and in CRA tissues, and revealed that $S N H G 22$ expression was upregulated in the order of ANTs $>$ CRA tissues $>$ TTs. High $S N H G 22$ expression levels were associated with advanced $\mathrm{T}$ stage, node involvement, metastasis and poor survival in patients with CRC. Therefore, $S N H G 22$ may serve as an independent prognostic indicator in patients with CRC.

As an oncogene, $S N H G 22$ may serve critical roles in tumor advancement processes; for example, it was shown to be highly expressed in EOC, and to stimulate EOC cell proliferation, invasion and chemotherapy resistance by sponging miR-2467 to facilitate galectin-1 expression in EOC cells (11). Fang et al (13) observed that, in breast cancer, SNHG22 facilitated tumor progression by sequestering miR-324-3p and subsequently upregulating SDS3 homolog, SIN3A corepressor complex component. The present study also examined the effects of SNHG22 on the phenotype of CRC cells, and revealed that SNHG22 promoted CRC cell proliferation, apoptosis resistance, invasion and migration in vitro. Knocking down $S N H G 22$ in vivo inhibited xenograft tumor growth. All these results implicate $S N H G 22$ as a possible critical oncogenic lncRNA in CRC.

Most cytoplasmic lncRNAs exert their regulatory effects on gene expression by functioning as ceRNAs, regulating miRNAs by competitively binding their target sites on protein-coding mRNA molecules $(25,26)$. For example, acting as a ceRNA, MIR17HG has been reported to base its biological behaviors on a sequence-specific interaction with miR-17-5p, thereby augmenting the biological roles of mRNA targets (5). Chen et al (27) reported that the lncRNA up-regulated in colorectal cancer liver metastasis promoted the invasion of CRC cell lines by sponging miR-215. Based on bioinformatics analyses and experimental assays, the present study demonstrated that SNHG22 was preferentially localized to the cytoplasm. Furthermore, it was confirmed that $S N H G 22$ could sponge miR-128-3p in CRC, and the dual-luciferase reporter assay indicated that SNHG22 may interact with miR-128-3p and hinder its biological roles. miR-128-3p has been reported to function as a tumor suppressor in several types of human cancer (28-32), and may be involved in cell proliferation, the cell cycle and chemosensitivity. It has been suggested that miR-128-3p may enhance the chemosensitivity of oxaliplatin-resistant CRC cells by targeting BMI1 proto-oncogene, polycomb ring finger and ATP-binding cassette subfamily C member 5 (30). Moreover, nanocomplexes loaded with miR-128-3p elevated chemotherapy roles through dual targeting, and silencing PI3K-AKT and MEK-ERK pathway activity in CRC cell lines (29).

As a ceRNA, the function of lncRNAs depends on the miRNA target gene. Using five online databases, E2F3 was predicted as a potential target of miR-128-3p. This hypothesis was validated with luciferase reporter assays. The results revealed that miR-128-3p overexpression inhibited the E2F3 wt 3 'UTR luciferase activity, but did not change that of the E2F3 mut 3'UTR. Furthermore, miR-128-3p overexpression inhibited E2F3 mRNA and protein expression levels, whereas silencing miR-128-3p elevated them, suggesting that $E 2 F 3$ 
could be a direct target of miR-128-3p in CRC cells. Further functional rescue experiments validated the hypothesis that $S N H G 22$ may regulate CRC proliferation and invasion by competitively sponging miR-128-3p and restoring $E 2 F 3$ activity. Recent studies have revealed that E2F3 may serve an essential role in regulating human cancer cell proliferation, apoptosis and chemosensitivity $(33,34)$.

In conclusion, the results of the present study revealed that, as an oncogenic lncRNA in CRC, $S N H G 22$ was upregulated and related to poor survival in patients with CRC. Functional and mechanistic analyses demonstrated that SNHG22 promoted CRC tumorigenesis and metastasis by sponging miR-128-3p, leading to elevated expression of E2F3. These findings may provide novel insights into the development of therapeutics for CRC.

\section{Acknowledgements}

Not applicable.

\section{Funding}

The present study was supported by the Key R \& D Promotion Projects of Henan Province 2019 (grant no. 192102310055).

\section{Availability of data and materials}

The datasets used and/or analyzed during the current study are available from the corresponding author on reasonable request or from the TCGA repository (https://portal.gdc.cancer.gov/.

\section{Authors' contributions}

LFZ, JNY, CFW and XYD conceived and designed the present study. JNY, YLL, HNZ and XYD performed the experiments. JNY, CFW, XYD and YZZ analyzed and interpretated the data. JNY, CFW and LFZ wrote, reviewed and/or revised the manuscript. JNY and LFZ confirm the authenticity of all the raw data. All authors read and approved the final manuscript.

\section{Ethics approval and consent to participate}

The present study involving human tissues was approved by the Ethics Committee of Zhengzhou University (approval no. 2011110402). All patients provided written informed consent prior to their inclusion within the study. The research has been carried out in accordance with the World Medical Association Declaration of Helsinki. All animal experiments were approved by the Committee of the Ethics of Animal Experiments of Zhengzhou University (approval no. 20180376).

\section{Patient consent for publication}

All patients included in the present study have provided consent for both participation and publication.

\section{Competing interests}

The authors declare that they have no competing interests.

\section{References}

1. Bray F, Ferlay J, Soerjomataram I, Siegel RL, Torre LA and Jemal A: Global cancer statistics 2018: GLOBOCAN estimates of incidence and mortality worldwide for 36 cancers in 185 countries. CA Cancer J Clin 68: 394-424, 2018.

2. De Angelis R, Sant M, Coleman MP, Francisci S, Baili P, Pierannunzio D, Trama A, Visser O, Brenner H, Ardanaz E, et al: Cancer survival in Europe 1999-2007 by country and age: Results of EUROCARE-5-a population-based study. Lancet Oncol 15: 23-34, 2014.

3. He Q, Long J, Yin Y, Li Y, Lei X, Li Z and Zhu W: Emerging roles of lncRNAs in the formation and progression of colorectal cancer. Front Oncol 9: 1542, 2020.

4. Luo Y, Yang J, Yu J, Liu X, Yu C, Hu J, Shi H and Ma X: Long non-coding RNAs: Emerging roles in the immunosuppressive tumor microenvironment. Front Oncol 10: 48, 2020.

5. Xu J, Meng Q, Li X, Yang H, Xu J, Gao N, Sun H, Wu S, Familiari G, Relucenti M, et al: Long noncoding RNA MIR17HG promotes colorectal cancer progression via miR-17-5p. Cancer Res 79: 4882-4895, 2019.

6. Xiao Z, Qu Z, Chen Z, Fang Z, Zhou K, Huang Z, Guo X and Zhang Y: LncRNA HOTAIR is a prognostic biomarker for the proliferation and chemoresistance of colorectal cancer via miR-203a-3p-mediated Wnt/B-catenin signaling pathway. Cell Physiol Biochem 46: 1275-1285, 2018.

7. Ding D, Li C, Zhao T, Li D, Yang L and Zhang B: LncRNA H19/miR-29b-3p/PGRN axis promoted epithelial-mesenchymal transition of colorectal cancer cells by acting on Wnt signaling. Mol Cells 41: 423-435, 2018.

8. Williams GT and Farzaneh F: Are snoRNAs and snoRNA host genes new players in cancer? Nat Rev Cancer 12: 84-88, 2012.

9. Shan Y, Ma J, Pan Y, Hu J, Liu B and Jia L: LncRNA SNHG7 sponges miR-216b to promote proliferation and liver metastasis of colorectal cancer through upregulating GALNT1. Cell Death Dis 9: 722, 2018

10. Li Y, Zeng C, Hu J, Pan Y, Shan Y, Liu B and Jia L: Long non-coding RNA-SNHG7 acts as a target of miR-34a to increase GALNT7 level and regulate PI3K/Akt/mTOR pathway in colorectal cancer progression. J Hematol Oncol 11: $89,2018$.

11. Ota T, Suzuki Y, Nishikawa T, Otsuki T, Sugiyama T, Irie R, Wakamatsu A, Hayashi K, Sato H, Nagai K, et al: Complete sequencing and characterization of 21,243 full-length human cDNAs. Nat Genet 36: 40-45, 2004.

12. Zhang PF, Wu J, Luo JH, Li KS, Wang F, Huang W, Wu Y, Gao SP, Zhang XM and Zhang PN: SNHG22 overexpression indicates poor prognosis and induces chemotherapy resistance via the miR-2467/Gal-1 signaling pathway in epithelial ovarian carcinoma. Aging (Albany NY) 11: 8204-8216, 2019.

13. Fang X, Zhang J, Li C, Liu J, Shi Z and Zhou P: Long non-coding RNA SNHG22 facilitates the malignant phenotypes in triple-negative breast cancer via sponging miR-324-3p and upregulating SUDS3. Cancer Cell Int 20: 252, 2020.

14. Gao H, Sun X, Wang H and Zheng Y: Long noncoding RNA SNHG22 increases ZEB1 expression via competitive binding with microRNA-429 to promote the malignant development of papillary thyroid cancer. Cell Cycle 19: 1186-1199, 2020.

15. Edge S, Byrd DR, Compton CC, Fritz AG, Greene F and Trotti A (eds): AJCC cancer staging handbook. 7th edition. Springer, New York, NY, 2010.

16. Livak KJ and Schmittgen TD: Analysis of relative gene expression data using real-time quantitative PCR and the 2(-Delta Delta C(T)) method. Methods 25: 402-408, 2001.

17. Du Y, Wei N, Hong J and Pan W: Long non-coding RNASNHG17 promotes the progression of breast cancer by sponging miR-124-3p. Cancer Cell Int 20: 40, 2020.

18. National Research Council (US) Committee for the Update of the Guide for the Care and Use of Laboratory Animals: Guide for the care and use of laboratory animals. 8th edition. National Academies Press (US), Washington, DC, 2011.

19. Cao Z, Pan X, Yang Y, Huang Y and Shen HB: The lncLocator: A subcellular localization predictor for long non-coding RNAs based on a stacked ensemble classifier. Bioinformatics 34: 2185-2194, 2018.

20. Lan Y, Xiao X, He Z, Luo Y, Wu C, Li L and Song X: Long noncoding RNA OCC-1 suppresses cell growth through destabilizing HuR protein in colorectal cancer. Nucleic Acids Res 46: 5809-5821, 2018. 
21. Wang Y, Lu JH, Wu QN, Jin Y, Wang DS, Chen YX, Liu J, Luo XJ, Meng Q, Pu HY, et al: LncRNA LINRIS stabilizes IGF2BP2 and promotes the aerobic glycolysis in colorectal cancer. Mol Cancer 18: 174, 2019.

22. Wang D, Zhang H, Fang X, Zhang X and Liu H: Prognostic value of long non-coding RNA GHET1 in cancers: A systematic review and meta-analysis. Cancer Cell Int 20: 109, 2020.

23. Xiao M, Feng Y, Liu C and Zhang Z: Prognostic values of long noncoding RNA PVT1 in various carcinomas: An updated systematic review and meta-analysis. Cell Prolif 51: e12519, 2018.

24. Yang W, Zhang K, Li L, Ma K, Hong B, Gong Y and Gong K: Discovery and validation of the prognostic value of the lncRNAs encoding snoRNAs in patients with clear cell renal cell carcinoma. Aging (Albany NY) 12: 4424-4444, 2020.

25. Li M, Bian Z, Jin G, Zhang J, Yao S, Feng Y, Wang X, Yin Y Fei B, You Q and Huang Z: LncRNA-SNHG15 enhances cell proliferation in colorectal cancer by inhibiting miR-338-3p Cancer Med 8: 2404-2413, 2019.

26. Yan J, Jia Y, Chen H, Chen W and Zhou X: Long non-coding RNA PXN-AS1 suppresses pancreatic cancer progression by acting as a competing endogenous RNA of miR-3064 to upregulate PIP4K2B expression. J Exp Clin Cancer Res 38: 390, 2019.

27. Chen DL, Lu YX, Zhang JX, Wei XL, Wang F, Zeng ZL, Pan ZZ, Yuan YF, Wang FH, Pelicano $\mathrm{H}$, et al: Long non-coding RNA UICLM promotes colorectal cancer liver metastasis by acting as a ceRNA for microRNA-215 to regulate ZEB2 expression. Theranostics 7: 4836-4849, 2017.

28. Zhao J, Li D and Fang L: MiR-128-3p suppresses breast cancer cellular progression via targeting LIMK1. Biomed Pharmacother 115: 108947, 2019.
29. Liu X, Dong C, Ma S, Wang Y, Lin T, Li Y, Yang S, Zhang W, Zhang R and Zhao G: Nanocomplexes loaded with miR-128-3p for enhancing chemotherapy effect of colorectal cancer through dual-targeting silence the activity of PI3K/AKT and MEK/ERK pathway. Drug Deliv 27: 323-333, 2020.

30. Liu T, Zhang X, Du L, Liu X, Tian H, Wang L, Li P, Zhao Y, Duan W, Xie Y, et al: Exosome-transmitted miR-128-3p increase chemosensitivity of oxaliplatin-resistant colorectal cancer. Mol Cancer 18: 43, 2019.

31. Li X, Lv X, Li Z, Li C, Li X, Xiao J, Liu B, Yang H and Zhang Y: Long noncoding RNA ASLNC07322 functions in VEGF-C expression regulated by smad4 during colon cancer metastasis. Mol Ther Nucleic Acids 18: 851-862, 2019.

32. Fu C, Li D, Zhang X, Liu N, Chi G and Jin X: LncRNA PVT1 facilitates tumorigenesis and progression of glioma via regulation of MiR-128-3p/GREM1 axis and BMP signaling pathway. Neurotherapeutics 15: 1139-1157, 2018.

33. Wang H, Wang L, Zhang S, Xu Z and Zhang G: Downregulation of LINC00665 confers decreased cell proliferation and invasion via the miR-138-5p/E2F3 signaling pathway in NSCLC. Biomed Pharmacother 127: 110214, 2020.

34. Chen J, Liu X, Xu Y, Zhang K, Huang J, Pan B, Chen D, Cui S, Song H, Wang R, et al: TFAP2C-activated MALAT1 modulates the chemoresistance of docetaxel-resistant lung adenocarcinoma cells. Mol Ther Nucleic Acids 14: 567-582, 2019. International (CC BY-NC-ND 4.0) License. 